\title{
Quality indicators of diabetes care: an example of remote-area Aboriginal primary health care over 10 years
}

\section{Julia V Marley \\ PgDipSc, PgDipPolSt, PhD, \\ Research Associate \\ Professor ${ }^{1,2}$ \\ Carmel Nelson \\ MPHandTM, FACRRM \\ FRACGP \\ Medical Director \\ Vicki O'Donnel \\ DipBusMgt \\ Chief Executive Officer \\ David Atkinson \\ MB BS, MPH, \\ Medical Coordinator,' and \\ Medical Educator ${ }^{2}$ \\ 1 Rural Clinical School of Western Australia University of \\ Western Australia \\ Broome, WA \\ 2 Kimberley Aboriginal Medical Services Council, Broome, WA \\ 3 Derby Aborigina Health Service Derby, WA. \\ Julia.Marley@ rcswa.edu.au}

MJA 2012; 197: 404-408 doi: 10.5694/mjal2.10275 nternational and Australian studies show that well structured approaches to diabetes care can improve short-term and intermediate patient outcomes. ${ }^{1,2}$ While there are several long-term randomised controlled trials aimed at improving intermediate outcomes in people with diabetes, ${ }^{3,4}$ there is only one published study on the long-term evaluation of a continuous quality improvement (CQI) program in realworld settings of primary health care. ${ }^{5}$ Ten years after this CQI program was introduced, there was significant improvement in intermediate outcomes and a reduction in microvascular and macrovascular disease in patients with diabetes in Catalonia. ${ }^{5}$

In Australia, only limited, generally short-term, studies have been published on the effectiveness of real-life service approaches to improving diabetes control. One showed significant improvement in some intermediate outcomes for patients with diabetes in Far North Queensland following the introduction of the "Meriba Zageth for diabetes" plan. ${ }^{6}$

Derby is a small remote Western Australian town with a harsh seasonal climate, $230 \mathrm{~km}$ from the regional centre of Broome and $2350 \mathrm{~km}$ from Perth tertiary referral centres. The 2006 census rated the Derby-West Kimberley area as the fifth most disadvantaged in Western Australia. ${ }^{7}$ Recruitment and retention of skilled professionals in Derby is a constant challenge, with a number of key local services characterised by high staff turnover. Derby Aboriginal Health Service (DAHS) is an Aboriginal community-controlled health service that was established in 1998. Key characteristics of DAHS include whole-ofservice involvement in internal CQI processes, use of electronic patient information and recall systems, and regional support and standardisation of care.

We aimed to report on long-term service activity and intermediate clini-

Objectives: To describe service characteristics of Derby Aboriginal Health Service (DAHS) and document diabetes management activities and intermediate clinical outcomes for Aboriginal patients with type 2 diabetes.

Design, setting and patients: Retrospective audit of records for patients $\geqslant 15$ years old who had a confirmed diagnosis of type 2 diabetes, received primary health care from DAHS for at least 6 continuous months between 1 July 1999 and 30 June 2009, resided in the Derby area and were not on renal replacement therapy.

Main outcome measures: Electronic records of blood pressure (BP), glycated haemoglobin $\left(\mathrm{HbA}_{\mathrm{lc}}\right)$ level, weight, albumin-creatinine ratio, creatinine level or estimated glomerular filtration rate, lipid levels and smoking status during each audit year; and proportions of patients who met clinical targets for $\mathrm{HbA}_{l c}$, BP and cholesterol.

Results: Over the 10 years, the proportion of clinical care activities undertaken according to regional protocols increased significantly, with very high levels recorded in the last 3 years (at least $70 \%$ of patients had each activity recorded). There were significant improvements in systolic BP, diastolic BP and cholesterol levels over the 10 years $(P<0.001$ for all). In the final year, $69 \%$ of patients had at least half their BP measurements $\leqslant 130 / 80 \mathrm{mmHg}$ and $83 \%$ had median annual cholesterol levels of $<5.5 \mathrm{mmol} / \mathrm{L}$. There were small improvements in $\mathrm{HbA}_{1 \mathrm{c}}$ levels that approached statistical significance $(P=0.05)$. In the final year, $34 \%$ of patients had median annual $\mathrm{HbA}_{1 \mathrm{c}}$ levels of $\leqslant 7.0 \%$.

Conclusions: This study shows that diabetes monitoring and outcomes can be improved and maintained over a 10-year period in a well supported remote Aboriginal community-controlled health service setting.

cal outcomes for DAHS Aboriginal and Torres Strait Islander patients with type 2 diabetes.

\section{Methods}

This study covers 10 audit years, from 1 July 1999 to 30 June 2009. It includes Aboriginal and Torres Strait Islander patients seen at DAHS who had type 2 diabetes mellitus, were $\geqslant 15$ years old, received primary care from DAHS for at least 6 continuous months, lived in or near Derby, and were not on renal replacement therapy.

The final study population was determined by carrying out a comprehensive retrospective review of paper and electronic records. During review of these records, the date of diagnosis of diabetes for each patient was confirmed as far as possible.

\section{Data extraction and cleaning}

In 2007, DAHS staff checked the accuracy of patients' electronic records against paper records. Results incorrectly recorded or not entered into Project Ferret (Pen Computer Systems), a patient information and recall system, were corrected. From 2007, data entry was monitored more closely. At the end of 2009, data were extracted from Project Ferret and transferred into Excel 2003 (Microsoft). Outliers were identified and data entry errors were corrected. Fewer electronic creatinine results than expected were observed during the audit years 2005-06 and 2006-07 and further corrections, based on paper records, were made. The data were then imported into Stata, version 11 (StataCorp).

\section{Service characteristics}

The major activities and factors likely to have had a direct impact on the quality of care provided to patients with diabetes at DAHS are listed in Box 1 .

\section{Quality indicators of diabetes care}

The occurrence of activities relating to diabetes management was assessed over the 10 audit years. Measures 
1 Major activities and factors likely to have directly affected quality of care provided by DAHS to patients with diabetes, 1999-2009

- Aboriginal community-controlled health service, with stable governance by community-elected board of directors and executive managers

- After operating in the early stages on a limited start-up budget, DAHS secured a purpose-built facility in 2005 and has drawn incrementally increasing resources to support service growth and diversification in response to demand

- Electronic health information systems:

$>$ in addition to paper records, a patient information and recall system (Project Ferret) was used to capture key clinical data; it was also used for reporting and CQI purposes

data entry clerk changed infrequently, ensuring consistency over time

- Significant consistency of senior staff

- Specialist outreach services, including visiting general physicians, dietitians, podiatrists and diabetes educators

- Formal and informal teaching and upskilling incorporated into routine practice: medical students hosted for their penultimate year, general practice registrar training, junior doctor placements, Aboriginal health worker student placements and nursing student placements

- CQI approaches based on a culture of organisational appraisal and improvement, encouraging review and reflection by staff at all levels, and embracing change in response to identified gaps

- Formalised CQI processes included:

brief participation in the Australian Primary Care Collaboratives program ${ }^{8}$ and the Audit and Best Practice for Chronic Disease project ${ }^{9}$

$>$ ongoing internal and external (Kimberley Aboriginal Medical Services Council) audit of service data

formalisation of regular audit process during the 2006-07 audit year, by introducing an internally developed standardised audit tool and routine audit process

- Regional support and standardisation of systems:

part of a regional collective of Aboriginal community-controlled health services > development and implementation across health sectors of the Kimberley Standard Drug List and evidence-based Kimberley Chronic Disease Therapeutic Protocols ${ }^{10}$

DAHS = Derby Aboriginal Health Service. $C Q \mathrm{I}$ = continuous quality improvement.

expected to be recorded at least 6 monthly (blood pressure [BP], glycated haemoglobin $\left[\mathrm{HbA}_{1 \mathrm{c}}\right]$ level, weight) were assessed over the last 6 months of each audit year (1 January to 30 June). Measures expected to be recorded annually (albumin-creatinine ratio $[A C R]$, creatinine level or estimated glomerular filtration rate [eGFR], lipid levels, smoking status) were assessed over the full audit year.

\section{Clinical outcome measures}

Clinical outcome measures were assessed each audit year. Overall median $\mathrm{HbA}_{1 \mathrm{c}}$ and cholesterol levels were determined using medians for individual patients during each audit year. Also, the proportions of patients with median $\mathrm{HbA}_{1 \mathrm{c}}$ and cholesterol levels meeting recommended targets ${ }^{11}$ were determined for each audit year. As the recommended target for BP includes meeting the target for both systolic $(\leqslant 130 \mathrm{mmHg})$ and diastolic ( $\leqslant 80 \mathrm{mmHg}$ ) measurements, the proportions of patients with at least half their electronic measurements meeting both targets were determined for each audit year.

Patients were classified as having proteinuria and/or kidney disease if they had at least two ACR results $>3.6 \mathrm{mg} / \mathrm{mmol}$ without obvious infection, creatinine or eGFR results suggestive of chronic kidney disease, ${ }^{10}$ and/or were assigned to a proteinuria or chronic kidney disease care plan.

\section{Statistical analysis}

A non-parametric test for trend across ordered groups was used to assess trend over time. ${ }^{12}$ Analyses were performed using Stata, version 12 (StataCorp). $P<0.05$ was considered statistically significant.

\section{Ethics approval}

Ethics approval was obtained from the Western Australian Aboriginal Health Information and Ethics Committee and exemption from ethics review was obtained from the Human Research Ethics Committee of the University of Western Australia.
Results

After identifying 517 patients with results suggestive of diabetes, 263 were excluded for not meeting the selection criteria (Box 2). Participants who commenced renal replacement therapy, stopped receiving primary care from DAHS or died were not included after these events occurred.

The final study population of 254 patients contributed a total of 1502 patient-years of care. Of this patient population, 107 (42\%) were diagnosed after becoming a regular patient at DAHS, 195 (77\%) regularly attended DAHS (as determined by the clinic) from when they entered the study until they were excluded or the study finished, 166 (65\%) were included in at least 5 audit years, 35 (14\%) were included in all 10 years and $45(18 \%)$ were included in both the first and last years. The median age of patients at the time of entering the study was 46 years (range, 15-84 years); 159 patients (63\%) were female, $185(73 \%)$ had or developed proteinuria and/or kidney disease, 12 (5\%) commenced renal replacement therapy and 41 (16\%) died.

Service activity for $\mathrm{BP}, \mathrm{HbA}_{1 \mathrm{c}^{\prime}}$ weight, ACR, creatinine level or eGFR, lipid levels and smoking status increased significantly over time $(P \leqslant 0.002$ for trend over 10 years; Box 3). Median annual systolic and diastolic BP values and cholesterol levels decreased significantly over time $(P<0.001$ for trend over 10 years; Box 4$)$. The proportion of patients who met currently recommended targets ${ }^{11}$ for median annual cholesterol and who had at least half their recorded results for $\mathrm{BP} \leqslant 130 / 80 \mathrm{mmHg}$ also significantly increased over time $(P<0.001$ for trend over 10 years; Box 4). Improvements in glycaemic control over time approached statistical significance $(P=0.05$ for trend over 10 years; Box 4). In the last year of the study, the proportions of patients who met the old targets of $\mathrm{HbA}_{1 \mathrm{c}}<7.0 \%$ and $\mathrm{BP}<130 / 80 \mathrm{mmHg}$ were $33 \%$ and 53\%, respectively. Previously reported data on care quality indicators and clinical outcome measures relating to diabetes care in other Australian populations are included in Box 3 and Box 4, respectively. 1,6,8,9,13-19 


\section{Selection process used to determine DAHS study population}

517 patients $\geqslant 15$ years old who had attended DAHS from 1 July 1999 to 30 June 2009 were identified as having results suggestive of diabetes, indicated by one or more of the following

- on a diabetes care plan

- diagnosis of diabetes on paper records

- taking hypoglycaemic medication

- fasting plasma glucose level $\geqslant 7.0 \mathrm{mmol} / \mathrm{L}$

- random plasma glucose level $\geqslant 11.1 \mathrm{mmol} / \mathrm{L}$

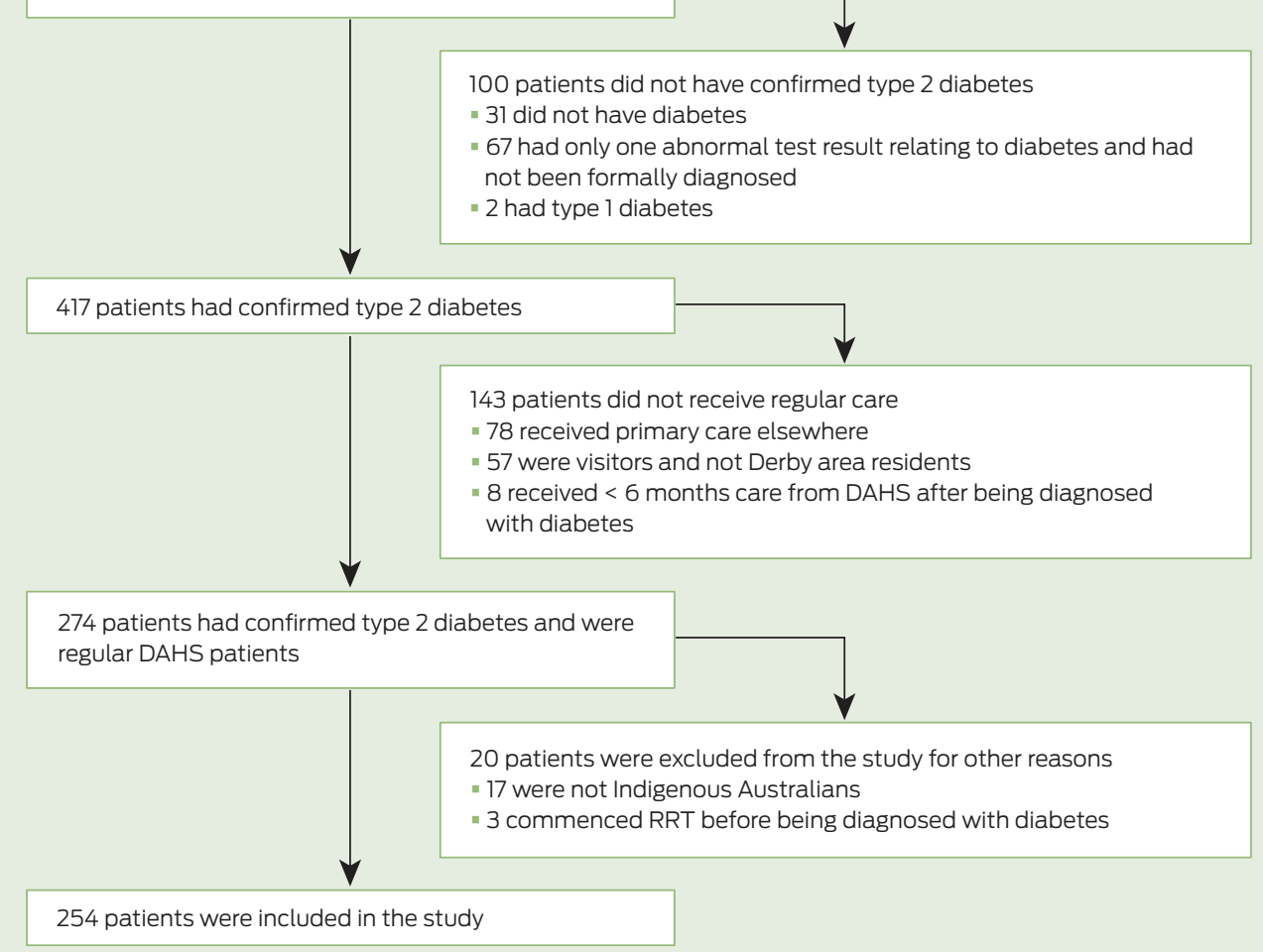

DAHS = Derby Aboriginal Health Service. $\mathrm{RRT}=$ renal replacement therapy .

\section{Discussion}

To our knowledge, this is the first published Australian study looking at 10 years of diabetes management in primary care at an individual health service. We showed significant and sustained improvements in health monitoring and intermediate outcomes at DAHS.

Improved care processes, improved control of BP and prevention of complications that result in hospitalisation have previously been demonstrated in Aboriginal community-controlled health services over short periods when adequate clinical support and appropriate management structures have been provided. ${ }^{2,13}$

The main strength of our study is that we used all recorded results for patients over a 10-year period and analysed clinical outcomes according to clinical targets from current guide- lines. ${ }^{11}$ Comparisons can be made with other studies, but caution is required because of the different methods and clinical targets used. The Australian Primary Care Collaboratives program operates through "waves" of 20-200 practices working together over 18 months, ${ }^{8}$ while the Audit and Best Practice for Chronic Disease project randomly selected 30 patients with diabetes from each of 62 services and used the most recent value for each patient within 12 months of the audit. ${ }^{9}$ The currently recommended target for $\mathrm{BP}$ is $\leqslant 130 /$ $80 \mathrm{mmHg}^{11}$ but previously it was $<130 / 80 \mathrm{mmHg}$. In our study, this meant that the proportion of patients with BP complying with target values changed from $53 \%$ to $69 \%$ in the final year. Similar changes occurred in the Australian National Diabetes Audit and Benchmarking project (54\% of patients with $\mathrm{BP} \leqslant 130 / 80 \mathrm{mmHg}$ in
2009 compared with $38 \%$ of patients with $\mathrm{BP}<130 / 80 \mathrm{mmHg}$ in 2006). ${ }^{17,20}$ The change in $\mathrm{HbA}_{1 \mathrm{c}}$ target $(<7.0 \%$ to $\leqslant 7.0 \%)$ made less difference in our study $(33 \% \mathrm{v} 34 \%$ of patients meeting the respective targets in the final year).

Limitations of this study included underrecording. In the earlier years, this may have contributed to the trend of improvements over time. As part of the CQI processes at DAHS, staff checked the accuracy of the measures reported in this article and updated electronic records, but some bias due to underrecording in the earlier years is likely. Our data on the duration of diabetes may also be biased because of difficulties in determining when diabetes was diagnosed in patients who were diagnosed at other health services. This study was also limited to data that were available; important outcomes, such as hospital admissions and medication usage, were not recorded electronically and more sophisticated measures relating to quality of life were not collected.

Our results compare well with those of other Australian studies of patients with diabetes. In terms of the intermediate outcome measures that we examined, BP was better controlled than in other Australian studies, including mainstream general practice studies $(69 \%$ v $28 \%-54 \% 8,16,17)$, and cholesterol levels were similar or better $\left(83 \%\right.$ v 53\%-86\% $\left.{ }^{9,15-19}\right)$. Glycaemic control was near the best reported in Indigenous Australian studies $\left(34 \%\right.$ v 22\%-32\% $\left.{ }^{1,6,9,14,15}\right)$ but not as good as has been reported in studies of mainstream general practice $(37 \%-57 \%) .8,18,19$

Despite the improvements we have shown, there is substantial room for further improvements in clinical outcomes. The focus on preventing or delaying kidney failure in the Kimberley may partly explain why BP control was particularly good in this study. Glycaemic control is considered more difficult to achieve compared with other measures ${ }^{6}$ and only small improvements in glycaemic control were evident in this study, although even small improvements can be clinically useful. Further investigation is planned to model $\mathrm{HbA}_{1 \mathrm{c}}$ measurements over time for individuals and determine factors that are likely to 
3 Proportions of DAHS study population for whom diabetes management activities were recorded in the electronic patient and information recall system during the 10 audit years, and corresponding values from the final years of other studies

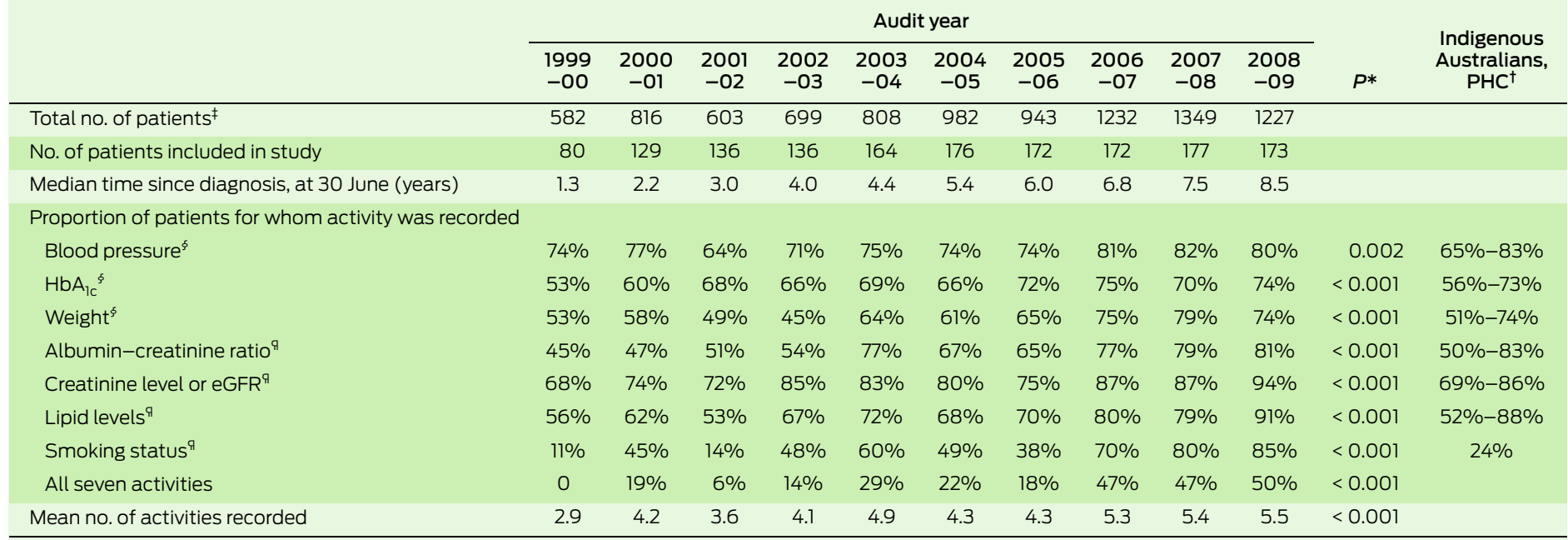

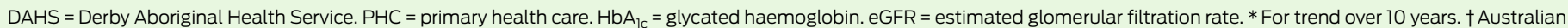

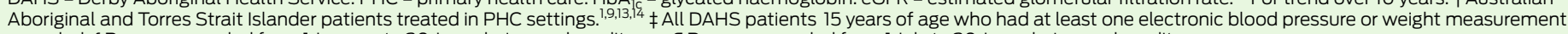
recorded. $\$$ Process recorded from 1 January to 30 June during each audit year. q Process recorded from 1 July to 30 June during each audit year.

contribute to individuals attaining and maintaining good glycaemic control.

From 2012, reporting of national key performance indicators (nKPIs) is a requirement for Aboriginal community-controlled health services under the National Indigenous Reform Agreement. ${ }^{21}$ The nKPIs include the $\mathrm{HbA}_{1 \mathrm{c}}$ and $\mathrm{BP}$ measures for patients with diabetes that we included in our study. It is hoped that the use of $n K P I s$ will result in comparable data from Aboriginal health services being produced.

Over the decade covered by our study, a number of policy changes were introduced at a national level which may have had an enabling role for DAHS in chronic disease care for its patients. These include the introduction of Medicare reimbursement for comprehensive Aboriginal and Torres Strait Islander preventive health checks and for chronic disease management planning and follow-up, ${ }^{22}$ and access to medications at low or no cost for Aboriginal people in remote areas (section 100 of the National Health Act 1953 [Cwlth]). ${ }^{23}$

DAHS had regular changes in health staff and management, but it also had significant consistency in senior staff over the decade we studied. The service also had improving resources over the study period, although resourcing started from a low base. DAHS maintained its community connections with a consistent board of directors, long-term employment of Aboriginal health workers and nurses, and a focus on genuine comprehensive primary health care for its patients.

Sustainability of chronic disease programs has previously been reported to be a major challenge in remote Aboriginal and Torres Strait Islander health settings. ${ }^{24}$ This study shows that diabetes monitoring and outcomes can be improved and maintained over a 10-year period in a remote Aboriginal community-controlled health service setting.

4 Clinical outcome measures of DAHS study population and proportions of those with outcome values that met currently recommended targets during the 10 audit years, and corresponding values from the final years of other studies

\begin{tabular}{|c|c|c|c|c|c|c|c|c|c|c|c|c|c|c|}
\hline & \multicolumn{10}{|c|}{ Audit year } & \multirow[b]{2}{*}{$P^{*}$} & \multirow{2}{*}{$\begin{array}{c}\text { Indigenous } \\
\text { Australians, } \\
\mathrm{PHC}^{\dagger}\end{array}$} & \multirow[b]{2}{*}{$\begin{array}{l}\text { Australians, } \\
\text { secondary }\end{array}$} & \multirow[b]{2}{*}{$\begin{array}{l}\text { Australians, } \\
\mathrm{PHC}^{\delta}\end{array}$} \\
\hline & $\begin{array}{l}1999 \\
-00\end{array}$ & $\begin{array}{c}2000 \\
-01\end{array}$ & $\begin{array}{l}2001 \\
-02\end{array}$ & $\begin{array}{c}2002 \\
-03\end{array}$ & $\begin{array}{l}2003 \\
-04\end{array}$ & $\begin{array}{c}2004 \\
-05\end{array}$ & $\begin{array}{c}2005 \\
-06\end{array}$ & $\begin{array}{c}2006 \\
-07\end{array}$ & $\begin{array}{l}2007 \\
-08\end{array}$ & $\begin{array}{c}2008 \\
-09\end{array}$ & & & & \\
\hline \multicolumn{15}{|c|}{ Median annual clinical outcome measures } \\
\hline Systolic BP (mmHg) & 135 & 134 & 130 & 128 & 124 & 120 & 120 & 120 & 120 & 120 & $<0.001$ & $128-130$ & 130 & 134 \\
\hline Diastolic BP (mmHg) & 85 & 80 & 81 & 80 & 80 & 80 & 78 & 75 & 74 & 75 & $<0.001$ & $78-80$ & 75 & 78 \\
\hline $\mathrm{HbA}_{\mathrm{lc}}(\%, \mathrm{mmol} / \mathrm{mol})$ & $8.2,66$ & $8.7,72$ & $8.2,66$ & $7.7,61$ & $8.1,65$ & $8.3,67$ & $8.1,65$ & $8.0,64$ & $7.6,60$ & $8.0,64$ & 0.05 & $8.1-9.0$ & 7.9 & $6.8-7.4$ \\
\hline Cholesterol (mmol/L) & 5.0 & 5.0 & 5.1 & 4.9 & 5.0 & 4.9 & 4.6 & 4.7 & 4.6 & 4.5 & $<0.001$ & $4.7-5.0$ & 4.3 & 4.7 \\
\hline \multicolumn{15}{|c|}{ Proportion of study population with median values that met targets } \\
\hline $\mathrm{BP} \leqslant 130 / 80 \mathrm{mmHg}$ & $38 \%$ & $46 \%$ & $44 \%$ & $53 \%$ & $66 \%$ & $66 \%$ & $67 \%$ & $75 \%$ & $70 \%$ & $69 \%$ & $<0.001$ & & $28 \%-54 \%$ & $34 \%$ \\
\hline $\mathrm{HbA}_{\mathrm{lc}} \leqslant 7.0 \%(\leqslant 53 \mathrm{mmol} / \mathrm{mol})$ & $26 \%$ & $28 \%$ & $34 \%$ & $34 \%$ & $31 \%$ & $24 \%$ & $32 \%$ & $36 \%$ & $39 \%$ & $34 \%$ & 0.05 & $22 \%-32 \%$ & $30 \%-32 \%$ & $37 \%-57 \%$ \\
\hline Cholesterol $<4.0 \mathrm{mmol} / \mathrm{L}^{* *}$ & $4 \%$ & $12 \%$ & $8 \%$ & $22 \%$ & $16 \%$ & $18 \%$ & $16 \%$ & $21 \%$ & $24 \%$ & $25 \%$ & $<0.001$ & $29 \%$ & & $22 \%$ \\
\hline Cholesterol $<5.5 \mathrm{mmol} / \mathrm{L}^{\dagger \dagger}$ & $64 \%$ & $63 \%$ & $71 \%$ & $76 \%$ & $73 \%$ & $72 \%$ & $76 \%$ & $75 \%$ & $81 \%$ & $83 \%$ & $<0.001$ & $65 \%-79 \%$ & $80 \%-86 \%$ & $53 \%-85 \%$ \\
\hline
\end{tabular}

DAHS = Derby Aboriginal Health Service. $\mathrm{PHC}=$ primary health care. $\mathrm{BP}=$ blood pressure. $\mathrm{HbA}_{\mathrm{lc}}=$ glycated haemoglobin. $*$ For trend over 10 years. $\dagger$ Australian Aboriginal and Torres Strait Islander patients treated in PHC settings. ${ }^{1,6,9,14,15} \neq$ General Australian population treated in secondary care settings. ${ }^{16,17} \oint \mathrm{General}$ Australian population treated in mainstream general practice settings. ${ }^{8,18,19}$ q At least half of each participant's recorded measurements met the recommended target for both systolic and diastolic BP.

** Recommended for people with diabetes. ${ }^{1} \dagger \dagger$ Recommended for the general population. 
Acknowledgements: This study was initiated and funded by DAHS as part of their COI program, with in-kind contributions including independent analysis by the Rural Clinical School of WA, at the University of Western Australia, and the Kimberley Aboriginal Medical Services Council. We had full access to all the data (including statistical reports and tables) in the study. We thank staff at DAHS for their efforts in cleaning up the database used in this study. We thank Max Bulsara and Sharon Evans for expert statistical advice and Sophie Couzos and lan Ring for critical reading of this article.

Competing interests: Carmel Nelson was the Senior Medical Officer (from 1999 to 2005) at DAHS. Vick O'Donnell is the Chief Executive Officer (from 2002 to present) of DAHS.

Received 9 Feb 2012, accepted 22 Aug 2012.

1 McDermott RA, Schmidt BA, Sinha A, Mills P. Improving diabetes care in the primary healthcare setting: a randomised cluster trial in remote Indigenous communities. Med J Aust 2001; 174: 497-502.

2 Si D, Bailie RS, Togni SJ, et al. Aboriginal health workers and diabetes care in remote community health centres: a mixed method analysis. Med $\rfloor$ Aust 2006; 185: 40-45.

3 Holman RR, Paul SK, Bethel MA, et al. 10-year follow-up of intensive glucose control in type 2 diabetes. NEngl J Med 2008; 359: 1577-1589.

4 Stratton IM, Adler Al, Neil HA, et al. Association of glycaemia with macrovascular and microvascular complications of type 2 diabetes (UKPDS 35): prospective observational study. BMJ 2000; 321: 405-412.

5 Mundet X, Cano F, Mata-Cases M, et al. Trends in chronic complications of type 2 diabetic patients from Spanish primary health care centres (GEDAPS study): ten year-implementation of St Vincent recommendations. Prim Care Diabetes 2012; 6: 11-18.

6 McDermott RA, McCulloch BG, Campbell SK, Young DM. Diabetes in the Torres Strait Islands of Australia: better clinical systems but significant increase in weight and other risk conditions among adults, 1999-2005. Med J Aust 2007; 186: 505-508.

7 Australian Bureau of Statistics, Australian Institute of Health and Welfare. The health and welfare of Australia's Aboriginal and Torres Strait Islander peoples 2005. Canberra: AlHW, 2005. (AlHW Cat. No. IHW 14.) http://www.aihw.gov. un/publication-detail/?id=6442467754 (accessed Aug 2012)

8 Ford DR, Knight AW. The Australian Primary Care Collaboratives: an Australian general practice success story. Med J Aust 2010; 193: 90-91.

9 Si D, Bailie R, Dowden M, et al. Assessing quality of diabetes care and its variation in Aboriginal community health centres in Australia. Diabetes Metab Res Rev 2010; 26: 464-473.

10 Kimberley Aboriginal Medical Services Council, WA Country Health Service Kimberley. Kimberley chronic disease protocols. 2010. http:// resources.kamsc.org.au/c_k_d_protocols.html (accessed Aug 2012).

11 Diabetes Australia, Royal Australian College of General Practitioners. Diabetes management in general practice. 17th ed. Melbourne: Diabetes Australia, 2011. http://www.racgp.org.au/ Content/NavigationMenu/ClinicalResources/ RACGPGuidelines/Diabetesmanagement/ 201107diabetesmanagementingeneralpractice. pdf (accessed Jun 2012).

12 Cuzick J.A Wilcoxon-type test for trend. Stat Med 1985; 4: 87-90.

13 McDermott R, Tulip F, Schmidt B, Sinha A Sustaining better diabetes care in remote Indigenous Australian communities. BMJ 2003; 327: 428-430.

14 Bailie RS, Si D, Robinson GW, et al. A multifaceted health-service intervention in remote Aboriginal communities: 3-year follow-up of the impact on diabetes care. Med J Aust 2004; 181: 195-200.

15 Maple-Brown L, Cunningham J, Dunne K, et al. Complications of diabetes in urban Indigenous Australians: the DRUID study. Diabetes Res Clin Pract 2008; 80: 455-462.
16 Bryant W, Greenfield JR, Chisholm DJ, Campbell LV. Diabetes guidelines: easier to preach than to practise? Med J Aust 2006; 185: 305-309.

17 National Association of Diabetes Centres. Australian national diabetes information audit and benchmarking: final report ANDIAB 2009. Canberra, 2009. http://www.health.gov.au/ internet/main/publishing.nsf/Content/pqdiabetes-pubs-andiab09 (accessed Sep 2011).

18 Kemp TM, Barr EL, Zimmet PZ, et al. Glucose, lipid, and blood pressure control in Australian adults with type 2 diabetes: the 1999-2000 AusDiab. Diabetes Care 2005; 28: 1490-1492.

19 Zwar NA, Hermiz O, Comino EJ, et al. Do multidisciplinary care plans result in better care for patients with type 2 diabetes? Aust Fam Physician 2007; 36: 85-89.

20 National Association of Diabetes Centres. Australian national diabetes information audit and benchmarking: final report ANDIAB 2006. Canberra, 2007. http://www.health.gov.au/ internet/main/publishing.nsf/Content/pqdiabetes-pubs-andiab06 (accessed Sep 2011).

21 Aboriginal Medical Services Alliance of the Northern Territory. OATSIH reporting reforms: reporting of national key performance indicators (nKPIs). 2011. http://www.amsant.org.au/ documents/article/94/factsheet5 reportingnKPIs.pdf (accessed Aug 2012).

22 Department of Health and Ageing. History of key MBS primary care initiatives 1999-2010. http:// www.health.gov.au/internet/main/publishing. nsf/Content/mbsprimarycare-History (accessed Jul 2012).

23 National Aboriginal Community Controlled Health Organisation. Pharmaceutical access. http://www.naccho.org.au/activities/ pharmaceutical.html (accessed Jul 2012).

24 Hoy WE, Kondalsamy-Chennakesavan SN, Nicol $J$. Clinical outcomes associated with changes in a chronic disease treatment program in an Australian Aboriginal community. Med J Aust 2005; 183: 305-309. 